\title{
Comparing the Binding Interactions in the Receptor Binding Domains of SARS-CoV-2 and SARS-CoV
}

\author{
Muhamed Amin,* Mariam K. Sorour, and Amal Kasry \\ Cite This: J. Phys. Chem. Lett. 2020, 11, 4897-4900 \\ Read Online
}

ABSTRACT: SARS-CoV-2, since emerging in Wuhan, China, has been a major concern because of its high infection rate and has left more than six million infected people around the world. Many studies endeavored to reveal the structure of the SARS-CoV-2 compared to the SARS-CoV, in order to find solutions to suppress this high infection rate. Some of these studies showed that the mutations in the SARS-CoV spike (S) protein might be responsible for its higher affinity to the ACE2 human cell receptor. In this work, we used molecular dynamics simulations and Monte Carlo sampling to compare the binding affinities of the $S$ proteins of SARS-CoV and SARS-CoV-2 to the ACE2. Our results show that the protein surface of the ACE2 at the receptor binding domain (RBD) exhibits negative electrostatic potential, while a positive potential is observed for the $S$ proteins of SARS-CoV/SARS-CoV-2. In addition, the binding energies at the interface are slightly higher for SARS-CoV-2 because of enhanced electrostatic interactions. The major contributions to the electrostatic binding energies result

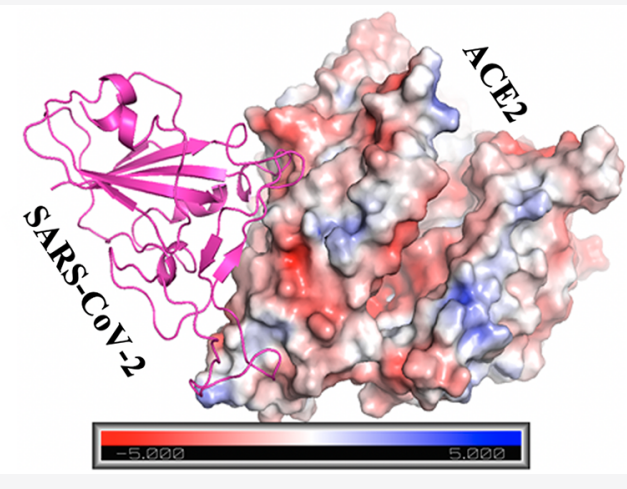
from the salt bridges forming between R426 and ACE-2-E329 in the case of SARS-CoV and K417 and ACE2-D30 in the SARSCoV-2. In addition, our results indicate that the enhancement in the binding energy is not due to a single mutant but rather because of the sophisticated structural changes induced by all these mutations together. This finding suggests that it is implausible for the SARS-CoV-2 to be a lab-engineered virus.

A highly infectious strain of coronavirus, known as SARSCoV-2, was first reported in Wuhan China on the 31st of December 2019. According to the World Health Organization (WHO), ${ }^{1}$ this strain has, as of May 29th, 2020, resulted in more than 5 million infections and over 300,000 deaths all over the world. Previous studies conducted on the first SARS-CoV strain showed that the most likely mechanisms by which the virus interacted with a cell were mediated by the receptor binding domain (RBD) on the spike protein (S protein), which binds with the peptidase domain of the human angiotensin-converting enzyme 2 (ACE2). ${ }^{2}$ Similarly, SARS$\mathrm{CoV}-2$ was proposed to enter the cells through the RBD. ${ }^{3-5}$ However, according to the latest resolved structure using Cryoelectronic microscopy, ${ }^{5}$ several mutations were identified in the RBD, which include V404 $\rightarrow$ K417, R426 $\rightarrow$ N439, Y442 $\rightarrow$ L455, L443 $\rightarrow$ F456, L460 $\rightarrow$ F473, L472 $\rightarrow$ F486, L479 $\rightarrow$ Q493, D480 $\rightarrow$ S456, Y484 $\rightarrow$ Q498, and T487 $\rightarrow$ N501. Furthermore, another study using comparative genomics identified only 5 mutations and stated that RBD does not contain insertion or deletion. ${ }^{6}$ The V404 $\rightarrow$ K417 mutation balances the R426 $\rightarrow$ N439, because they can cause addition and removal of the positively charged amino acids, respectively. Moreover, the D480 $\rightarrow$ S456 mutation removes a negatively charged amino acid and is, therefore, expected to make the electrostatic surface potential of SARS-CoV-2 more positive.
In this work, we study the binding interaction between the amino acids on the interface between the ACE2 receptor and the $S$ protein of SARS-CoV and SARS-CoV-2 viruses using molecular dynamics (MD) simulations and Monte Carlo (MC) sampling.

The crystal structure $\mathrm{PDB}$ ID $2 \mathrm{AJF}^{7}$ which includes the receptor binding domain $\mathrm{RBD}$ of SARS-CoV and the ACE2, was first MD optimized using openMM software, ${ }^{8-13}$ followed by running Monte Carlo simulations using MCCE (Multi Conformer Continuum Electrostatic) software, ${ }^{14,15}$ to sample the protonation states of the amino acids. The electrostatic interactions between the different amino acids' conformers were calculated by solving the Poisson-Boltzmann equation using DELPHI. ${ }^{16}$ The generated conformers' occupancies based on Boltzmann distributions were used to calculate the electrostatic and van der Waals interactions between the amino acids in the SARS-CoV and the ACE2.

Received: April 4, 2020

Accepted: June 1, 2020

Published: June 1, 2020 
To study the interactions between the SARS-CoV-2 and the ACE-2, the mutants V404K, R426N, Y442L, L443F, F460Y, L472F, N479Q, D480S, Y484Q and T487N were constructed based on the cryoEM structure PDB ID $6 \mathrm{M} 17 .{ }^{7}$ The simulations were performed by replacing the side chains in the native structures with the proper side chains of the mutants using MCCE. Several confirmations of the side chains were created to avoid van der Waals clashes. The side chain conformers with the highest Boltzmann occupancies were then MD optimized, followed by using MCCE to calculate the binding energies.

The electrostatic potential maps of SARS-CoV, SARS-CoV2, and ACE2 were calculated using Adaptive PoissonBoltzmann Solver (APBS). ${ }^{15}$ While both SARS-CoV and SARS-CoV-2 showed positive potentials (Figure 1a,b), ACE2

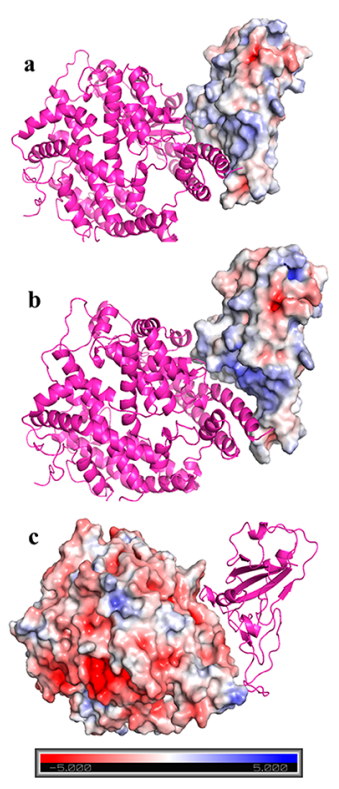

Figure 1. Electrostatic potential maps (in kT/e) of (a) the SARS$\mathrm{CoV}$, (b) SARS-CoV2, and (c) ACE2 (SAR-CoV is shown in a cartoon view). The ACE2 is shown in cartoon view in panels $a$ and $b$.

exhibited a negative electrostatic potential at the RBD, as shown in Figure $1 \mathrm{c}$. However, the potential observed for SARS-CoV-2 was more positive, which consequently resulted in a greater electrostatic interaction between ACE2 and SARSCoV-2. This could be explained by the replacement of the negatively charged amino acid Asp by a neutral Ser (D480S).

The optimized structure of SARS-CoV showed a salt bridge between SARS-CoV-R426 and ACE2-E329 (Figure 2a), which dominated the electrostatic interactions with the ACE2. In the case of SARS-CoV-2, the corresponding salt bridge was shown to be forming between CoV-2-K404 and ACE2-D30 (Figure 2b). However, the latter exhibited a higher electrostatic interaction by $1.4 \mathrm{kcal} / \mathrm{mol}$ (Table 1 ). The contribution in the free energy formation of the salt bridges $(\Delta G)$ is dominated by the pariwise acid/base interactions (Table S4).

The total electrostatic interactions between the SARS-CoV2 and the ACE- 2 is $3 \mathrm{kcal} / \mathrm{mol}$ higher than SARS-CoV (Table $\mathrm{S} 1)$. The contribution of a single mutant to the electrostatic binding energies is very small (Table S1). However, these mutants induce structural changes that increase the favorable van der Waals interactions in SARS-CoV-2.

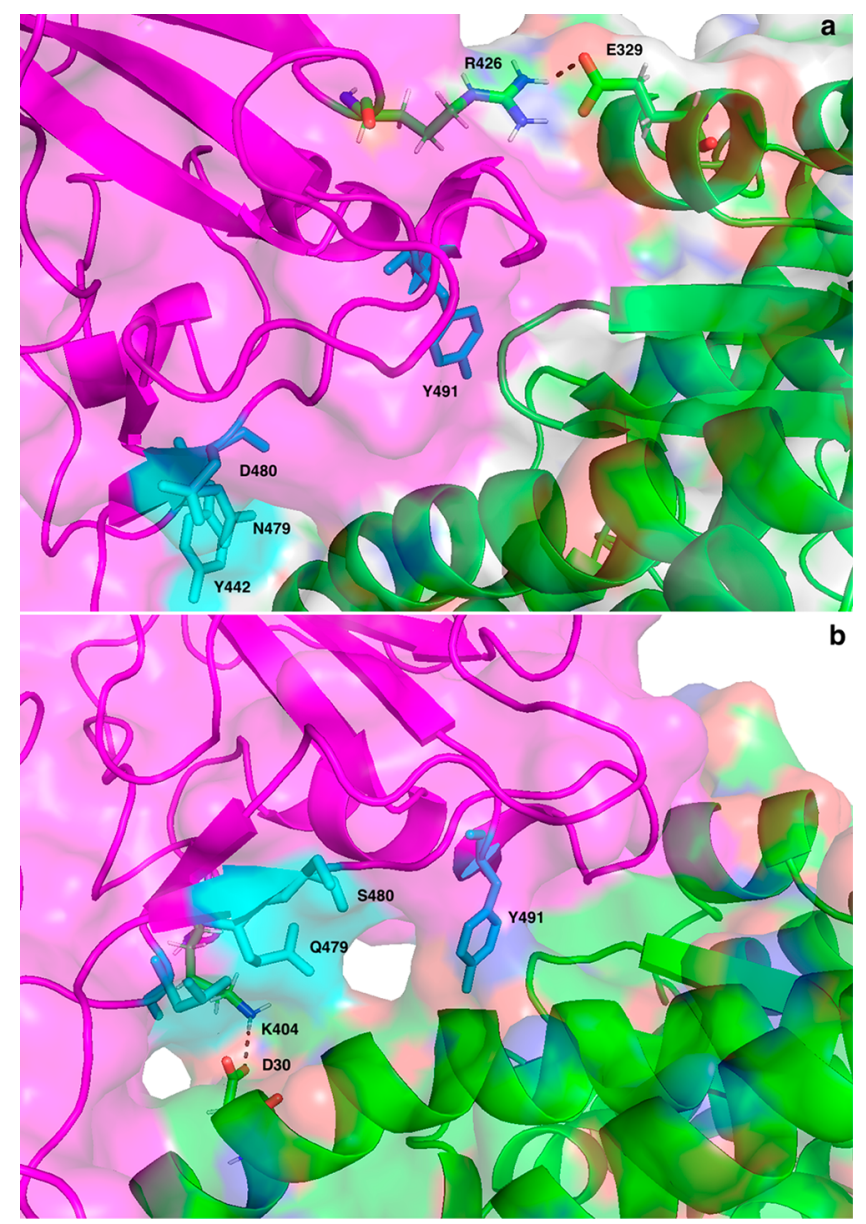

Figure 2. MD optimized structures of (a) SARS-CoV and (b) SARS$\mathrm{CoV}-2$. The secondary structures of the $S$ protein and the ACE2 are shown in magenta and green, respectively. Key residues are labeled and shown in cyan. The salt bridges formed between R426 and E329 in SARS-CoV and K404 and D30 in SARS-CoV-2 are shown as black dots. The transparent surface is presented to show the solventaccessible surface. Table S3 shows the surface-accessible areas of the amino acids.

Table 1. Total Electrostatic and van der Waals Interactions between SARS-CoV, SARS-CoV-2, and ACE-2 ${ }^{a}$

$\begin{array}{rlrl}\text { CoV } & \rightarrow \text { ACE-2 } & \text { CoV-2 } & \rightarrow \text { ACE- } 2 \\ \text { R426 } & \rightarrow \text { E329, }-5.83 & \mathrm{~K} 404 & \rightarrow \mathrm{D} 30,-7.22 \\ \mathrm{~K} 390 & \rightarrow \mathrm{D} 37,-1.01 & \mathrm{Y} 491 & \rightarrow \mathrm{E} 37,-1.28 \\ \mathrm{D} 393 & \rightarrow \mathrm{K} 353,-0.95 & \mathrm{~K} 390 & \rightarrow \mathrm{D} 37,-1.21 \\ & & \text { van der Waals }(\mathrm{kcal} / \mathrm{mol}) \\ \mathrm{Y} 475 & \rightarrow \mathrm{T} 27,-1.56 & \mathrm{D} 491 \rightarrow \mathrm{K} 353,-2.10 \\ \mathrm{I} 489 & \rightarrow \mathrm{Q} 325,-1.34 & \mathrm{Y} 475 \rightarrow \mathrm{K} 31,-1.50\end{array}$

${ }^{a_{T}}$ The major electrostatic contributions are observed for the R426/ E329 salt bridge for SARS-CoV and K404/D30 for SARS-CoV-2. Table S1 includes more information about the electrostatic and van der Waals interactions between SARS-CoV, SARS-CoV-2, and ACE2.

A maximum van der Waals interaction of $1.6 \mathrm{kcal} / \mathrm{mol}$ was observed between SARS-CoV-Y457 and ACE2-T27, whereas for SARS-CoV-2 the maximum van der Waals interaction observed between CoV2-D491 and ACE2-K353 was $2.1 \mathrm{kcal} /$ mol (Table 1). The total van der Waals contribution to the binding energy was $4 \mathrm{kcal} / \mathrm{mol}$. The total sum of binding 
interactions between the SARS-CoV-2 and the ACE2 receptor was found to be $\sim 7 \mathrm{kcal} / \mathrm{mol}$, which is higher than the total binding energy in the case of SARS-CoV by a factor of 1.54 .

To obtain a better sampling we calculated the electrostatic interactions between SARS-CoV/SARS-CoV-2 and the ACE2 for different $\mathrm{MD}$ trajectories at 100,200 , and 300 ps. The binding energies for SARS-CoV/SARS-CoV-2 are comparable. However, on average the SARS-CoV-2 binds stronger than the SARS-CoV (Table S2).

Furthermore, to illustrate the overall structural changes in the S-protein in SARS-CoV-2, we calculated the binding energies starting from the cryo-Em structure resolved for the Sprotein of SARS-CoV-2 and the ACE2 (PDB ID: 6M17). We found that the total binding energy in the case of SARS-CoV-2 is stronger, where the electrostatic and van der Waals interactions are 3 and $1 \mathrm{kcal} / \mathrm{mol}$, respectively, higher than in case of the SARS-CoV.

Several studies proposed that the increased virulence of SARS-CoV-2 is due to its higher binding affinity to the ACE2 receptor. $^{3,6,7}$ Yan et al. proposed that mutation V404 $\rightarrow$ K417 may result in higher binding affinity due to the salt bridge between K417 and D30, whereas the R426 $\rightarrow$ N439 mutation would weaken the interaction with E329. ${ }^{7}$ However, our simulations show that the V404 $\rightarrow$ K417 mutation increased the binding affinities because of favorable electrostatic interactions that are greater than the energy losses induced by R426 $\rightarrow$ N439 mutation. Additionally, mutation L472 $\rightarrow$ F486 was proposed to weaken van der Waals contact with Met 82 of ACE2. In contrast, the relaxed structure showed a van der Waals attraction of $-1.32 \mathrm{kcal} / \mathrm{mol}^{7}$ The structural changes induced by these mutations likely conferred stability to SARS-CoV-2 as it binds to ACE2. This agrees with the study of Ortega et al., ${ }^{17}$ in which they found that two main residues (479 and 487) have been associated with human ACE2 recognition. They further found a higher number of residues in the SARS-CoV-2 capping loops and attributed the more favorable binding affinity of $-15.7 \mathrm{kcal} / \mathrm{mol}$ as opposed to $-14.1 \mathrm{kcal} / \mathrm{mol}$ for SARS-CoV. ${ }^{17}$ Our results also agree with the experimental work of Wallas et al, where they studied the binding kinetics and affinity of the purified human ACE2 to both SARS-CoV-2 and SARS-CoV. They found that the equilibrium dissociation constant in the case of the first is $\sim 4$ times lower than the second, indicating much higher affinity between SARS-CoV-2 and ACE2. ${ }^{18}$

In summary, we show that the total binding energy between ACE2 and SARS-CoV-2 is higher than in the case of SARS$\mathrm{CoV}$, because of enhanced electrostatic interactions induced by the SARS-CoV-2 mutations. Based on our calculations, there is no significant contribution from a single mutant to the binding energies; however, these mutations induce sophisticated structural changes that enhance the electrostatic and van der Waals binding energies. These results might also support the idea that it is unlikely that the SARS-CoV-2 is lab engineered, but rather a result of a biological evolution. ${ }^{19}$

\section{ASSOCIATED CONTENT}

\section{(s) Supporting Information}

The Supporting Information is available free of charge at https://pubs.acs.org/doi/10.1021/acs.jpclett.0c01064.

Detailed description of the MCCE and MD calculations, Table $S 1$ of interactions between amino acids within the RBD, Table S2 of the electrostatic and van der Waals interaction for different MD trajectories, and Table S3 of the surface exposure of key amino acids, Table S4 of the $\Delta G$ 's of the ACE2-D30/SARS-CoV-R426 and ACE2E329/SARS-CoV-2-K404 salt bridges and the coordinates of the relaxed SARS-CoV-2 structures (PDF)

\section{AUTHOR INFORMATION}

\section{Corresponding Author}

Muhamed Amin - Department of Sciences, University College Groningen, University of Groningen, 9718 BG Groningen, The Netherlands; Center for Free-Electron Laser Science, Deutsches Elektronen-Synchrotron DESY, 22607 Hamburg, Germany; Centre for Theoretical Physics, The British University in Egypt,

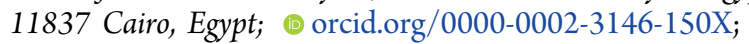
Email: m.a.a.amin@rug.nl

\section{Authors}

Mariam K. Sorour - Nanotechnology Research Centre (NTRC), the British University in Egypt (BUE), Cairo 1183, Egypt

Amal Kasry - Nanotechnology Research Centre (NTRC), the British University in Egypt (BUE), Cairo 1183, Egypt; (i) orcid.org/0000-0002-8130-8693

Complete contact information is available at:

https://pubs.acs.org/10.1021/acs.jpclett.0c01064

\section{Author Contributions}

M.A. proposed the idea and ran the MC and MD simulations. M.K.S. and A.K. worked on collecting the recent work published related to $\mathrm{CoV}-19$ to find out the relevant differences between SARS-CoV and SARS-CoV-2, in order to define the mutations. All authors contributed to different aspects of the work, including writing and reviewing the Letter.

Notes

The authors declare no competing financial interest.

\section{ACKNOWLEDGMENTS}

This work is supported by the University of Groningen and the Nanotechnology Research Centre (NTRC), the British University in Egypt (BUE). We acknowledge the funding from NSF MCB 1519640 grant to M.R.G.. We thank Prof. Marilyn Gunner and Prof. Jochen Kupper for useful discussions.

\section{REFERENCES}

(1) Rolling updates on coronavirus disease (COVID-19), Events as They Happen; World Health Organization Website.

(2) Li, F.; Li, W.; Farzan, M.; Harrison, S. C. Structure of SARS Coronavirus spike Receptor-Binding Domain Complexed with Receptor. Science 2005, 309, 1864-1868.

(3) Walls, A. C.; Park, Y. J.; Tortorici, M. A.; Wall, A.; McGuire, A. T.; Veesler, D. Structure, Function, and Antigenicity of the SARSCoV-2 spike Glycoprotein. Cell 2020, 181, 281-292.

(4) Wrapp, D.; Wang, N.; Corbett, K. S.; Goldsmith, J. A.; Hsieh, C. L.; et al. Cryo-EM Structure of the 2019-NCoV spike in the Prefusion Conformation. Science 2020, 367, 1260-1263.

(5) Ou, X.; Liu, Y.; Lei, X.; Li, L.; Mi, D.; et al. Characterization of spike glycoprotein of SARS-CoV-2 on virus entry and its immune cross-reactivity with SARS-CoV. Nat. Commun. 2020, 11, 1620.

(6) Wan, Y.; Shang, J.; Graham, R.; Baric, R. S.; Li, F. Receptor Recognition by the Novel Coronavirus from Wuhan: An Analysis Based on Decade-Long Structural Studies of SARS Coronavirus. J. Virol. 2020, 94, e00127-20. 
(7) Yan, R.; Zhang, Y.; Li, Y.; Xia, L.; Guo, Y.; Zhou, Q. Structural Basis for the Recognition of SARS-CoV-2 by Full-Length Human ACE2. Science 2020, 367, 1444-1448.

(8) Friedrichs, M. S.; Eastman, P.; Vaidyanathan, W.; Houston, M.; LeGrand, S.; et al. Accelerating Molecular Dynamic Simulation on Graphics Processing Units. J. Comput. Chem. 2009, 30, 864-872.

(9) Eastman, P.; Pande, V. S. OpenMM: A Hardware-Independent Framework for Molecular Simulations. Comput. Sci. Eng. 2010, 12, 34-39.

(10) Eastman, P.; Pande, V. S. Constant Constraint Matrix Approximation: A Robust, Parallelizable Constraint Method for Molecular Simulations. J. Chem. Theory Comput. 2010, 6, 434-437.

(11) Eastman, P.; Pande, V. S. Efficient Nonbonded Interactions for Molecular Dynamics on a Graphics Processing Unit. J. Comput. Chem. 2010, 31, 1268-1272.

(12) Eastman, P.; Friedrichs, M. S.; Chodera, J. D.; Radmer, R. J.; Buns, C. M.; et al. OpenMM 4: A Reusable, Extensible, Hardware Independent Library for High Performance Molecular Simulation. J. Chem. Theory Comput. 2013, 9, 461-469.

(13) Eastman, P.; Swails, J.; Chodera, J. D.; McGibbon, R. T.; Zhao, Y.; et al. OpenMM 7: Rapid development of high-performance algorithms for molecular dynamics. PLoS Comput. Biol. 2017, 13, e1005659.

(14) Song, Y.; Mao, J.; Gunner, M. R. MCCE2: Improving protein pKa calculations with extensive side chain rotamer sampling. $J$. Comput. Chem. 2009, 30, 2231-2247.

(15) Jurrus, E.; Engel, D.; Star, K.; Monson, K.; Brandi, J.; et al. Improvements to the APBS biomolecular solvation software suite. Protein Sci. 2018, 27, 112-128.

(16) Teixeira, S.; Pacheco, X.; Borland Delphi 6 developer's guide; Sams Publishing, 2002.

(17) Ortega, J. T.; Serrano, M. L.; Pujol, F. H.; Rangel, H. R. Role of changes in SARS-CoV-2 spike protein in the interaction with the human ACE2 receptor: An insilico analysis. EXCLI J. 2020, 19, 410417.

(18) Walls, A. C.; Park, Y. J.; Tortorici, M. A.; Wall, A.; McGuire, A. T.; Veesler, D. Structure, Function, and Antigenicity of the SARSCoV- 2 Spike Glycoprotein. Cell 2020, 181, 281-292.

(19) Andersen, K. G.; Rambaut, A.; Lipkin, W. I.; Holmes, E. C.; Garry, R. F. The proximal origin of SARS-CoV-2. Nat. Med. 2020, 26, 450-452. 